\title{
The viability of utilising phone-based text messages in data capture and reporting of morbidities due to Lymphatic Filariasis by Community Health Workers: A qualitative study in Kilwa district, Tanzania
}

Akili Kalinga ( $\square$ kalingaaka@yahoo.com )

National Institute for Medical Research (NIMR)

Michael Munga

National Institute for Medical Research (NIMR)

Abdallah Ngenya

National Institute for Medical Research (NIMR)

Winfrida John

National Institute for Medical Research (NIMR)

William Kisoka

National Institute for Medical Research (NIMR)

Ndekya Oriyo

National Institute for Medical Research (NIMR)

Prince Mutalemwa

National Institute for Medical Research (NIMR)

Wilfred Mandara

National Institute for Medical Research (NIMR)

Leonard Masagati

National Institute for Medical Research (NIMR)

John Ogondiek

National Institute for Medical Research (NIMR)

Patricia Korir

Institute for Medical Microbiology, Immunology and Parasitology (IMMIP), Bonn

Ute Klarmann-Schulz

Institute for Medical Microbiology, Immunology and Parasitology (IMMIP), Bonn

Sacha Horn

University Hospital Munich, Ludwig-Maximilians- Universität (LMU)

Inge Kroidl

University Hospital Munich, Ludwig-Maximilians- Universität (LMU)

Alex Debrah

Kwame Nkrumah University of Science and Technology (KNUST) 


\section{Achim Hoerauf}

Institute for Medical Microbiology, Immunology and Parasitology (IMMIP), Bonn

\section{Upendo Mwingira}

National Institute for Medical Research (NIMR)

\section{Research Article}

Keywords: Mobile phone-based text messages, mobile technology, Community Health Workers, Lymphatic Filariasis, morbidity

Posted Date: November 18th, 2021

DOI: https://doi.org/10.21203/rs.3.rs-1074243/v1

License: (c) (i) This work is licensed under a Creative Commons Attribution 4.0 International License.

Read Full License 


\section{Abstract \\ Background}

Globally, there is recognition of the value of using mobile phones among health providers in improving health systems performance. However, in many Low- and Middle-income countries where there is shortage of health providers, Community Health Workers have assumed some responsibilities especially relating to identifying and reporting on health problems within their communities. Despite the known benefits of using mobile phone technology to deliver health services, there is limited information on the extent to which Community Health Workers are able to effectively use the technology in data collection and reporting. The aim of this study was to determine the feasibility of utilizing phone-based text messages on Lymphatic Filariasis morbidity surveillance by Community Health Workers

\section{Methods}

This was a cross sectional study whose data was collected through key informant interviews and focused group discussions among community health workers, health providers and staff of neglected tropical diseases control program. Collected transcripts were analysed through Thematic content analysis as it allowed for the identification of data codes through inductive reasoning.

\section{Results}

The use of mobile phone-based text messages in data collection and reporting lymphatic filariasis morbidity cases by Community Health Workers was perceived by study participants to be a relevant tool and feasible due to the ease of use of the technology. The tool was reported by end-users to significantly increase their performance and efficiency was gained in terms of reduced paper work, increased the number of patients accessing health care services and the ability to report in real-time. All respondents were confident that Community Health Workers were the right persons to interact with communities in tracking and reporting morbidity cases using mobile technology.

\section{Conclusion}

Mobile phone-based text messages have proven to be effective in routine workflows such as, data collection and reporting on Lymphatic Filariasis morbidity cases, patient to provider communication, decision making and supportive supervision. Mobile phones have also improved efficiency and general performance of end users in terms of increased number of cases identified and efficiency gained in terms of reduced paper work and the ability to collect and report in real-time.

\section{Background}


Lymphatic filariasis (LF) is one of the most common disabling neglected tropical diseases (NTDs) targeted for elimination globally $(1,2)$ and in Tanzania (3).Worldwide, around 51 million people are reported to be affected with $\operatorname{LF}(2)$.

Globally, it is estimated that 40 million people suffer from LF related morbidities with only 34 out of 73 endemic countries actively implementing morbidity management and disability prevention (MMDP) services(4). Lymphoedema is managed through hygiene and skin care to prevent episodes of adenolymphangitis, wound care, exercise, elevation of the affected limb and proper footwear $(5,6)$. Hydrocele can be managed through lymphatic draining or hydrocelectomy (7).

The WHO Road map (2021-2030) for neglected tropical diseases (NTDs) set a target of eliminating LF as a public health problem in order to achieve sustainable Developmental Goals (SDG) through feasible, cost-effective approaches $(8,9)$. For a country to be declared free of LF it has to enumerate people with LF morbidities and provide care to those patients. That is, endemic countries are recommended to provide minimum care for hydrocele patients and lymphoedema patients of different stages (10). Worldwide there are only seventeen countries including three countries from Africa which have been validated to eliminate LF as a public health problem (11).

Therefore, even if the transmission of the infection is largely stopped, there remains the issue of disease sequelae, such as lymphedema and hydrocele

Globally, it is estimated that 40 million people suffer from LF related morbidities with only 34 out of 73 endemic countries actively implementing morbidity management and disability prevention (MMDP) services (4). Lymphoedema is managed through hygiene and skin care, wound care, exercise, elevation of the affected limb and proper footwear to prevent episodes of adenolymphangitis $(5,6)$. Hydrocele can be managed through lymphatic draining or hydrocelectomy (7).

Tanzania adopted the Global Program to Eliminate Lymphatic Filariasis (GPELF) in 2000 under the Lymphatic Filariasis Elimination Program (LFEP). LFEP focuses on giving anti-filarial drugs to all eligible people in the endemic areas via mass drug administration (MDA) and provide management and prevent disability among those already having LF-morbidities $(12,13)$. Although major achievements have been made in terms of interrupting LF transmission in 111 (93.3\%) out of 119 filarial endemic districts in Tanzania, the management of LF major chronic manifestations (lymphoedema and hydrocele) has not been paid enough attention (14). From 2017 to 2020, only a total of 6658 surgeries of hydrocele have been conducted countrywide while about 1134 people with lymphoedema have been trained and supported with hygiene kits to manage their conditions(14). Unfortunately, there is no exact number of people suffering from both LE and hydrocele countrywide; it therefore, becomes difficult to calculate the proportion of individuals with LF morbidities.

The management of lymphoedema requires sustainable surveillance systems to track cases due to LF morbidities from remote areas where the disease is prevalent in order for them to access available care. 
In Tanzania, like many other endemic developing countries, there is a lack of a robust surveillance system to track cases of LF morbidities, especially in hard-to-reach areas (15).

The increased use of mobile phone technology has been shown to enhance the health systems in resource limited settings by improving data collection and reporting $(16,17)$.Additionally, there is growing evidence pointing to the effectiveness of using mobile phone technology to increase the effectiveness of Community Health Workers ( $\mathrm{CHWs}$ ) in delivering services to the community members while at the same time improve the efficiency of data collection and quality of data $(16,18,19)$. Real time data collection and reporting of LF morbidity through mobile phone technology can be an essential resource in the LF management value chain(20). The use of mobile phone technology to report LF morbidity cases was piloted by CHWs in Kilwa District, Lindi region, Dar es Salaam and Mtwara region using a text-based short messaging service (SMS) tool, $(16,21)$. This approach has helped generate a close-to accurate estimate of LF burden data but has not yet been scaled up for nationwide use due to many factors including overreliance on paper-based data collection and reporting, technical difficulties among health providers, and lack of understanding of the service delivery $(22,23)$.

\section{Community Health Workers: who are they and what are they doing?}

The term "Community Health Workers" (CHWs) is used to describe a diversity of health aides who are selected and trained to work in their own communities. CHWs can be men or women, young or old, literate or illiterate (24). Moreover, CHWs ensure community acceptance and ownership of existing health services (25). Preventive chemotherapy for the roles and activities of CHWs are enormously diverse, within and across countries and across programs(26). While in some cases $\mathrm{CHWs}$ perform a wide range of different tasks that can be preventive, curative and/or developmental, in other cases $\mathrm{CHWs}$ are appointed for very specific interventions $(27,28)$.

In Tanzania, in the context of supporting LFEP under the Neglected Tropical Diseases Control Program (NTDCP), CHWs have often been referred to as Community Drug Distributors (CDDs), a name that got fame initially from Community directed treatment with Ivermectin (CDTI) for control of Onchocerciasis and later with for five NTDs preventive chemotherapy using MDA(29). This emphasizes that the definition of $\mathrm{CHWs}$ must be contextualized to respond to local societal and cultural norms and customs to ensure community acceptance and ownership(30). In this study, CHWs were assessed for the feasibility of using them in identifying from their localities, managing and reporting LF morbidity cases using mobile phonebased text messages.

\section{The Context}

The healthcare sector in Tanzania relies largely on manual, paper-based patient records that are not centralised or integrated, creating communication barriers for different actors in the healthcare system. Within the LFEP, the routine activities of case identification, data collection, and reporting are done in collaboration with $\mathrm{CHWs}$ by health providers at primary level health facilities. The morbidity burden of LF in Tanzania has relied on the information from the MDA program where CHWs passively report cases 
identified annually at their localities. Consequently, the exact prevalence of morbidity cases has not always been accurate at the community or national level $(31,32)$.

Literature has shown the usefulness of mobile phones in improving health systems performance increasing efficiencies and effectiveness in health care delivery in many low-income countries. In general, evidence shows that receiving text messages via mobile phones improves patients' adherence to antiretroviral therapy and other clinical outcomes $(16,18)$.

Despite the known benefits of using mobile phone technology, there is limited information, in the context of NTDs, on whether CHWs are able to effectively use it to report the implementation of activities at the community level and thus establish a base for monitoring program effectiveness. The aim of this study was to determine the feasibility of phone-based text message on LF morbidity surveillance system using $\mathrm{CHWs}$ for the patients to access MMDP services as one of the few attempts seeking to bridge this information gap.

\section{Materials And Methods}

\section{Study area}

The study was conducted in Kilwa district in Lindi region, located in the south east of Tanzania. Kilwa district was purposely selected to have due to documented high large number of LF cases (personal communication with NTD control program manager) and is one of the major hotspot areas for LF transmission in the country (NTDCP, 2017).

\section{Study design}

This was a cross sectional study which employed a qualitative method of data collection and analysis. The study was conducted between December, 2019 and January, 2020.

\section{Study population}

The study population for this study were CHWs, representative LF patients and health providers working with NTDCP. The CHWs who initially participated in a study conducted in the same communities, to identify, manage and report to the national level, LF morbidities using mobile phone-based text messages were thought to be the right participants of this study to give perceptions on their involvement in NTDCP surveillance work using tested digital health technology and assess the feasibility of future use of this strategy. LF morbidity cases who were tracked in the communities under this study who were reported through MPBTMs were also realized as study population. Both male and female of all age who were health providers working with NTDCP at district and national levels were considered suitable participants of this study.

\section{Selection of study respondents}


The key informants who mostly were health providers were chosen purposefully to benefit from their knowledge based on their strategic positions in LF case identification, provision of MMDP services and those involved in managerial role of LF control programmes at national and district levels. Participants of focus group discussions (FGDs) were also purposively selected among LF morbidity cases and CHWs who were residing in same communities and participated the sister study.

\section{Data collection methods}

Key informant interviews (KIIs) and FGDs were held to assess the feasibility of LF morbidity surveillance by using phone-based text messaging as a data collection and surveillance tool by engaging $\mathrm{CHWs}$. In this study a total of 11 FGDs and 16 KIls were conducted. KII and FGD guides were used to collect responses from the participants during interviews and discussions respectively. The guides comprised a number of issues for discussions regarding $\mathrm{CHWs}$ perceptions, experiences and challenges faced in using mobile phones-based text messages as data collection and reporting tool, and their perceived effectiveness in identifying LF cases and morbidity management, among others. Both guides for KII and FGD were pre-tested prior to data collection. The guides were later adjusted and refined based on the feedback from pre-testing and all interviewers and FGD moderators were thoroughly trained by the experienced research team members on all important technical and ethical aspects of conducting qualitative research. All KIls and FGDS were conducted in Swahili which is the national official language spoken by more than 80 percent of the Tanzanian populations. Each KII and FGD session took between 45 minutes and one hour at a place and time that was most convenient to the interviewee and discussants respectively.

\section{Data analysis:}

Qualitative data were analysed iteratively. All voice recordings from FGDs and interviews were transcribed and translated into English by research assistants. To check for accuracy, $10 \%$ of the transcripts were back translated into Swahili. Members of the research team then compared Kiswahili and English versions for differences and similarities while listening to the original voice recordings. Content and thematic analysis of transcripts followed transcription, allowing for the identification of data codes through inductive reasoning. The analysis commenced with the coding of transcripts, then organized and sorted by relevant themes for reporting. Sub-themes were also identified in the data through an iterative process, and codes were refined as needed during the analysis. Written notes from interviews complemented the transcripts. Data triangulation and verification occurred by comparing responses from different sources to identify similar themes and areas of (dis)agreement on issues across data sources and participants/respondents. An inductive approach to data analysis was used, guided by four questions based on the four pre-determined themes, namely: 1) perceived benefits of training prior to starting using mobile phones 2) CHWs/CDDs perspectives on their effectiveness in using mobile phones as data capturing and reporting tool 3) challenges faced by CHWs/CDDs in using mobile phones as data collection and reporting tool 4) Effects of training (if any) on using mobile phone technology as a data 
collection and reporting tool. Additional themes that emerged during analysis are also reported in the results section of this paper.

\section{Results}

\section{The effect of training of CHWs in enhancing data collection and reporting}

It is important to note that before starting using mobile phones as a data collection and reporting tool, $\mathrm{CHWs}$ were engaged in training on how to use mobile phones as a tool to support data collection and reporting on LF morbidities. This section presents their experiences and perceived benefits of training prior to starting using mobile phones, perceptions on the feasibility, effectiveness and perceived challenges of using phone-based text messages in data collection and reporting LF morbidities.

It was almost unanimously conceded by KIs and majority of participants of the FGD sessions that the training helped the beneficiaries reduce errors in data collection and reporting hence enriched decision making at higher levels of the NTDCP. This was done by improving all aspects of care for the cases, including safety, cleanliness, communication and adherence. In fact, many further admitted that the training conducted helped CHWs, and those in the NTD control program to effectively and efficiently full fill their responsibilities and better on understanding procedures the patients should be doing to manage their conditions. CHWs themselves reported that training improved their approaches to communicate with communities and followed required steps especially when managing LE cases at community level. NTDCP officials reported on their perspective that through training CHWs were able to track and identify individuals with morbidities due to LF. As such, there was an increased number of patients reported at national level. Therefore, training has increased effectiveness in reporting and managing of LF Cases:

"I can say the training was beneficial to us CHWs and to our patients. Prior to training CHWs, LF patients used to hide in their homes or farms. But after we have been trained and used our knowledge to sensitize and educate our patients on how to manage their conditions and where to get treatment, they have changed and became receptive of the service offered by us and now they are coming out public" (FGD, Mandawa)

Additionally, it was conceded by majority of FGD participants that stigma-reducing messages through sensitization and health education given to community has made some patients accept their conditions and start seeking medical services something which was initially seen as unusual practice in the past.

\section{Effectiveness and feasibility of using mobile-based LF morbidity surveillance system.}

Apart from discarding the need for them to carry manual registers during field visits, $\mathrm{CHWs}$ perceived a greater level of efficiency in routine reporting and record keeping especially when mobile phones were used albeit in tandem with manual registers. Generally, the use of mobile phones was perceived to be relevant and thus feasible for the day-to-day activities of $\mathrm{CHWs}$. Further perception amongst $\mathrm{CHWs}$ is that 
the mobile phone-based text messages enabled a more direct channel of communication with senior health staff at the district and national NTDCP level concerning LF morbidity cases.

... "I think it is relevant and acceptable because it has simplified the reporting work, it is faster than paperbased reporting and the good thing these days almost everybody in the community has a mobile phone..." (Female FGD participant, Mandawa).

"Communication is everything. The world has been reduced to a village because people communicate easily, so is the case with our situation" (FGD, Female, Somanga)

Compared to paper-based data collection and reporting the mobile phone- based system was perceived to be more effective.

"When reporting using papers, sometimes papers can get lost or torn especially when it is raining but with mobile phones it is easy and comfortable...." (Female FGD participant, Kilwa Masoko)

According to the respondents from both FGDs, the mobile phone technology has the potential to enhance community engagement through encouraging and simplifying the exchange of information between lower and higher levels of health program. Among the mostly cited advantages that were mentioned are real-time reporting of LF morbidity cases and enhanced CHWs-patient interactions. Respondents viewed the technology to be simple, fast and effective.

.... "By using the Phone-based text messages for LF morbidity surveillance, an instant message of the information required is received and what is most important is the availability of stable network" (Male FGD participant, Mandawa).

Another participant had to say

... "It is easy to use the menu in mobile phone because it is similar in fastness and familiar to one used for sending money and it only needs signals for mobile phone company to send text messages" (CDD Miteja).

It was learnt from both FGDs and KIIs that mobile phones are instrumental in facilitating referrals of the identified patients to available health care services for morbidity management and remotely providing health education. The observation below further illustrates:

... "Mobile phones have narrowed the communication gap between the CHWs and LF clients on the one hand, and between CHWs and health providers on the other. Generally, CHWs had a perception that their effectiveness in case identification, referrals and provision of health education for morbidity management for LF patients has increased as a result of using mobile phone technology". (Male FGD participant/CHWs, Tingi).

Another participant responded that 
... "My experience with mobile phones, especially after being trained to use it for data collection and reporting has, to a large extent, made my job easier and I can reach and connect clients to health services faster than before. I can remotely provide health education to the people I serve in the community and I can monitor progress of their conditions even without coming into direct physical contact with them". (Female FGD participant CHWs, Miteja)

\section{Perceived impact of using mobile phones-based text messages on performance of CHWs}

The use of mobile phone-based text messages was said to enhance LF morbidity cases' follow up by CHWs. That is, the ability to track patients and their information and transmit them to national NTDCP level was enhanced and thus leading to improved performance. Generally, Many CHWs viewed the use of mobile phone-based text messages in monitoring and reporting cases as very crucial in improving the performance of health care staff. Through monitoring and reporting cases, staffs are able to notice the trend of LF morbidity cases in their respective communities.

"The phone helps in bringing patients information faster, and it helps knowing how many new patients we have at the community. And after receiving patient information we handle it promptly and effectively it will help to control and reduce LF disease" (NTDCP officer, Miteja).

Using mobile phone technology was further perceived as an effective tool for reaching many patients in a short time regardless of how remote and sparsely located they are.

... "The mobile phone-based data collection and reporting system by CHWs helped in easy of reaching more LF patients. Getting LF patient without using this system is a huge undertaking because as I mentioned earlier, LF is a common disease that someone develops and stays with it all his/her life in hard-to-reach areas. We have, through campaign, sensitized communities to view this as a common disease which can be managed in health facilities. But by using text messaging system they can report the disease through $\mathrm{CHWs}$ by using mobile phones without having national teams or facility staffs of $L F$ coordinators to go looking for them. So, using phones is a great innovation in not only dealing with the existing LF morbidities but also in registering new cases"(NTDCP officer, Kilwa district council).

"It will help. For example, new cases or even old cases the CHW will be aware of the trend, whether according to the services he/she provides patients are decreasing or vice versa, all these will be in the reporting trend" (NTDCP officer, Kilwa district council).

The use of mobile phones among CHWs was perceived by majority of FGD participants to be associated with increased access to health care services and coverage of population. Health education provided by CHWs has brought up patients who would otherwise hide in their homes as a result of stigma or misconceptions about the disease and all its manifestations.

... "using mobile phones has helped us to easily identify LF morbidity patients and understand different stages of the disease progression. We do not end at identifying and staging the patients but we also refer them to the health facilities for further action." (Male FGD participant, Mandawa) 


\section{Perceived contribution of CHWs in reduction of LF morbidity and transmission of disease}

Positive perceptions by community members were demonstrated towards $\mathrm{CHWs}$ as having an important contribution in tracking LF morbidity cases for them to access MMDP services provided jointly by NTDCP and partners. Additionally, CHWs are involved in MDA activities at community level that contributes to reduction of transmission of the disease. Therefore, their role in As such, affected people thought that by CHWs involved also in distributing ivermectin to a community, a job often done by CHWs, contributes to curtailing disease transmission within their localities. One discussant put it:

"In my opinion because they give us drugs (Ivermectin through MDA) and insist that we should use bed nets consistently, that is the contribution of CHWs in transmission control" (FGD, Adult Male, Tingi)

Another discussant observed that since CHWs started using mobile phones, there has been noticeable improvement in the management of their conditions. This was attributed to the ease of communication and reporting.

"I think when they (CHWs) report our situation to higher authorities in time, those people respond immediately and we get attention accordingly" (FGD adult Female, Tingi)

\section{Perceived challenges experienced during case identification and reporting using mobile phone-based text messages}

The experienced challenges can be understood from three interrelated perspectives, namely; those of the LF patients, CHWs, and of district and national program officials. Note that, the critical challenges are those perceived by CHWs who are the primary users of the digital technology in data collection and reporting of the LF morbidity cases as described here.

Despite popular perceptions by $\mathrm{CHWs}$ on the advantages of using mobile phones in data capturing and reporting, concerns were also raised regarding associated challenges related to using mobile phone technology. The frequently cited challenge by the majority of KIls and FGD participants was the mobile phone's network instability especially in very remote areas.

.... "One of the challenges which is not within our reach is network connection breakdown. Although this is not so frequent but it happens some time when you are submitting a report to the program officers at the national level; you may think it has gone but it takes time to reach the destination or sometimes it is not delivered altogether" (FGD male Participant, Miteja)

It was further revealed that electricity was also critical for the smooth functioning of mobile phones. For mobile phones that need to be regularly charged, power black-out was frequently mentioned by the majority of FGD participants as a challenge they face in using mobile phones for data capturing and reporting. 
... "Not every village has a stable electricity supply and network coverage. Hence if that happens, CHWs may not be able to send the information to recipients until the systems stabilizes, this becomes a critical set back. Issues mostly experienced are calls not received, the system not returning responses, phones not charged and poor communication system. All these experiences make the text messaging system some of the times to be counterproductive". (FGD male Participant, Mandawa)

.... "Unreliability of electricity is quite challenging in our contexts. Sometimes one is forced to wait for 8 to 12 hours' power black-out before power normalizes again. So, this makes it difficult to collect patients' data and report them timely to the required authorities (FGD Female participant, Kinyonga)

It was further learnt that the challenges that affect the LF clients and CHWs at the community level have a spill over effects on the operations of facility health staff at the primary health care level, district NTDCP program officials and finally affect the amount and quality of data captured at the national LF data repository. Accordingly, one informant revealed this:

... "The NTDCP implements its activities using a cascaded bottom-up approach. Many operations are implemented from the bottom by CHWS in close collaboration with the local community leaders, incharges of primary health care facilities and community members. From there, information is sent to the district level and in these days with the help of mobile phones, information can as well be sent directly to the national program level. The national level provides program/policy guidance, Standard operating procedures (SOPS), capacity building and M\&E. So, definitely all the challenges that affect operations at the lower level will definitely affect the data that is captured at the national level". (NTDCP officer, Kilwa district council).

\section{Discussion}

The importance of including end users' perspectives for effective implementation of mHealth interventions (e.g. mobile-phone based text messages as analysed in this study) has been emphasized in the past(33) however, the contribution of qualitative evaluations of the use of mobile phones to supplement the implementation of many mHealth interventions has not received much attention in many Low and Middle Income Countries (LMIC). This study adds important insights by presenting the CHWs' (here in referred to as end-users of technology) perspectives on the effectiveness of mobile phone-based text message in data collection and reporting morbidity due to LF in a rural endemic district of Tanzania. In addition, the paper discusses on CHWs' perceptions of mobile phone-based text messages in improving their effectiveness in managing their day-to-day responsibilities in supporting the NTDCP in Tanzania.

Findings of this study have shown that CHWs have a positive perception that the uses of mobile phonebased text messages increase their effectiveness and efficiency in routine reporting and record keeping of LF morbidities even when mobile phones are used in tandem with manual registers. CHWs have thus endorsed phone-based text messages in their day-to-day activities as useful and relevant. In line with the above findings, it is reported that CHWs in Uganda and Mozambique also felt that using mobile phone 
technology had the potential to improve their work efficiency, planning, and communication with supervisors(34).

Some of the encouraging results highlighted in this study include improvement of service delivery planning, time management and efficiency, accuracy of collected data, communication with caregivers, caregiver's compliance with instructions, individual increased CHWs' performance and accountability, and simplification of the work routine. This finding is in line with what has been reported in other studies(16, $19,35)$. Accuracy of the collected data is hereby emphasized as there were strong and positive perceptions by KIls and FGD members that, compared to paper-based data collection, phone-based text messages for data collection has big potential in minimizing errors, it is user friendly and efficient in terms of saving time because information are shared in real-time (36).

The findings of this study corroborate the global literature and further point out that the use of mobile phone technology by $\mathrm{CHWs}$ has improved outreach services, data collection and management, and effectiveness in the reporting of morbidities $(35,37)$. Moreover, mobile phone technologies are believed to have the potential to improve users' knowledge, skills, and performance as reported elsewhere $(16,38-$ 40). Perceptions of CHWs in this study have also confirmed this revelation especially when they relate to the training; they received prior to using mobile phones as a tool for data collection and reporting of LF morbidity cases.

Generally, the use of mobile technology in the delivery of health services, especially in remote settings where poverty related diseases are prevalent and where there is shortage of professional health providers is recommended for simplification of work, increasing accuracy and performance of staff who works for professional staff through task shifting. The use of mobile phone technology has proven effective and is associated with increased performance in various countries in Africa namely Ghana(41), Uganda (42) and Indonesia (43). In Tanzania, the study evaluated the impact of short message service (SMS) reminders to improve $\mathrm{CHW}$ promptness of $\mathrm{CHW}$ visits. The study found that the escalating reminder system is very beneficial in which the SMS reminder is sent directly to the CHW before notifying the CHW's supervisor. The intervention resulted in an $86 \%$ reduction in the number of days a CHW's routine visits were overdue (9.7 to 1.4 days)(44).

In this study, it was acknowledged by the majority of KIls and members of the FGD sessions that the training provided helped the beneficiaries to reduce errors in data collection and reporting and thus enhanced decision making at the higher levels of the program. This was done by improving all aspects of care for the cases, including safety, hygiene, communication and adherence. Many further admitted that the training helped $\mathrm{CHWs}$ to effectively and efficiently fulfil their responsibilities.

Despite the positively perceived advantages of using mobile phone-based text message as demonstrated in this study, the innovation cannot go without implementation challenges. One of the mostly cited challenges was weak mobile signals which affected communication and unreliability of electricity was also repeatedly mentioned as a challenge as phones need to be sufficiently charged for all the mobile phone operations to go on smoothly. 
A number of studies suggest that $\mathrm{CHWs}$ encounter various challenges when using mobile health solutions such as mobile phones for health service delivery. These include lack of CHWs training on new mHealth solutions, weak technical support, issues of weak signal strength and other administrative related challenges. A study conducted in Rwanda found that regular training of frontline health workers reduced the error rate for data entry from $54 \%$ at the start of the program to $8 \%$ over the course of 1 year(45). Thus, it is important to provide sufficient initial and ongoing training to support the transition of workflow from a paper-based system to a digitized system(46). The findings presented in this paper especially those relate to challenges of phone charging due to unreliability of electricity from frequent power black-out and poor network coverage that were faced by $\mathrm{CHWs}$ agrees with the Ugandan and the Mozambican formative study (34).

\section{Conclusions}

Mobile phone-based text messages tool used by CHWs has proven to be effective in their routine workflows such as, data collection and reporting on Lymphatic Filariasis morbidity cases, patient to provider communication, decision making and supportive supervision. The tool has also improved efficiency and general performance of CHWs in terms of increased number of cases identified, gains by reducing paper work and the ability to collect and report in real-time. The applicability and importance of the findings of this study should extend beyond the Kilwa district in Tanzania to similar settings in the country and other LMIC.

\section{List Of Abbreviations}

BMBF Bundesministerium für Bildung und Forschung

CDD Community Drug Distributors (CDD)

CDTI Community Directed Treatment with Ivermectin

IMMIP Institute for Medical Microbiology, Immunology and Parasitology

KNUST Kwame Nkrumah University of Science and Technology

RTI Research Triangle Institute

MDA Mass Drug Distributors

GPELF Global Program to Eliminate Lymphatic Filariasis

LFEP Lymphatic Filariasis Elimination Program

NTDs Neglected Tropical Diseases

NTDCP Neglected Tropical Diseases Control Program 
WHO World Health Organization

CHWs Community Health Workers

NIMR National Institute for Medical Research

\section{Declarations}

\section{Ethical approval and consent to participate}

The study was granted ethical clearance from national Medical Research Coordinating Committee (MRCC) with approval certificate number NIMR/HQ/R.8a/Vol.IX/2607. Additionally, permission to conduct the study was sought from Regional and District authorities including Regional Medical Officer (RMO) for the Lindi region and District Medical Officer (DMO) for the Kilwa district respectively. Permission was also sought from community leaders to engage their CHWs and LF morbidity cases in the study. A thorough explanation was given to participants about the aims and re-assurance that participation was voluntary and that a participant was free to withdraw from the study or terminate participation at any time they wished so. An informed consent was sought from participants after explaining the aims of the study.

\section{Consent for publication}

Not applicable.

\section{Availability of data and materials}

The datasets analyzed from this study for presentation of the results and concluding about the study are available on special request from $r$ the corresponding author

\section{Competing interests}

There are no competing interests in this study among authors

\section{Funding}

This study was funded by German Federal Ministry of Education and Research (Bundesministerium für Bildung und Forschung - BMBF); As the host of the project, the National Institute for Medical Research (NIMR) co-supported the projects through their own contribution to the project.

\section{Authors' contributions}

$A K, A N, U M$ and $A D$ designed the study; WJ, MM, WK, LM, JO \&PM drafted the manuscript. MM, WK, AK \&PM contributed to data analysis. AKK, MM, AH, AD, WK, UM, PJ, UK, IK, SH, AH and PM critically revised the manuscript. All authors read and approved the final version of the manuscript submitted herewith for publication 


\section{Acknowledgements}

Authors are very thankful to the German Federal Ministry of Education and Research (Bundesministerium für Bildung und Forschung - BMBF) for funding this study. The management of National Institute for Medical Research (NIMR) is acknowledged for hosting the study and administrative procedures during the implementation of study. We are grateful to the South African Centre for Infectious Diseases Surveillance (SACIDS) for their permission to use their portal in data transfer through phone USSD system using a government code $\left({ }^{*} 152^{\star} 00 \#\right)$. Many thanks are extended to Research Assistants who interviewed study participants and prepared notes and transcripts. Finally, we are indebted to the CHWs, patients and health providers to their voluntary participation in the study.

\section{Authors' information}

Not applicable

\section{References}

1. de Souza DK, Gass K, Otchere J, Htet YM, Asiedu O, Marfo B, et al. Review of MDA registers for lymphatic filariasis: Findings, and potential uses in addressing the endgame elimination challenges. PLoS Negl Trop Dis. 2020;

2. Deshpande A, Miller-Petrie MK, Johnson KB, Abdoli A, Abrigo MRM, Adekanmbi V, et al. The global distribution of lymphatic filariasis, 2000-18: a geospatial analysis. Lancet Glob Heal. 2020;8(9):e1186-94.

3. Lupenza E, Gasarasi DB, Minzi OM. Lymphatic filariasis, infection status in Culex quinquefasciatus and Anopheles species after six rounds of mass drug administration in Masasi District, Tanzania. Infect Dis Poverty [Internet]. 2021;10(1):1-11. Available from: https://doi.org/10.1186/s40249-02100808-5

4. Chandrasena N, Premaratna R, Gunaratna IE, de Silva NR. Morbidity management and disability prevention for lymphatic filariasis in Sri Lanka: Current status and future prospects. PLoS Negl Trop Dis. 2018;

5. Stocks ME, Freeman M, Addiss D, Stocks ME, Freeman MC, Addiss DG. The Effect of Hygiene-Based Lymphedema Management in Lymphatic Filariasis-Endemic Areas: A Systematic Review and Metaanalysis Copyright information : The Effect of Hygiene-Based Lymphedema Management in Lymphatic Filariasis-Endemic Areas : A Systematic. 2019;0-19.

6. H. N, M. M, M. N, J.A. B, E. K, P. N, et al. Lymphoedema management to prevent acute dermatolymphangioadenitis in podoconiosis in northern Ethiopia (GoLBeT): a pragmatic randomised controlled trial. Lancet Glob Heal. 2018;

7. Chandrasena N, Premaratna R, Gunaratna IE, de Silva NR. Morbidity management and disability prevention for lymphatic filariasis in Sri Lanka: Current status and future prospects. PLoS Negl Trop Dis. 2018 May;12(5):e0006472. 
8. Soc TR, Med T. A road map for neglected tropical diseases 2021 - 2030. 2021;121-3.

9. Turner HC, Bettis AA, Chu BK, McFarland DA, Hooper PJ, Ottesen EA, et al. The health and economic benefits of the global programme to eliminate lymphatic filariasis (2000-2014). Infect Dis Poverty. 2016;

10. WHO. Morbidity management and disability prevention in lymphatic filariasis. 2013;

11. WHO. Ending the neglect to attain the Sustainable Development Goals: a road map for neglected tropical diseases 2021-2030. Geneva: World Health Organization (https://www.who.int/neglected_diseases/Revised-DraftNTD-Roadmap-23Apr2020.pdf). 2020;196.

12. Derua YA, Kisinza WN, Simonsen PE. Lymphatic filariasis control in Tanzania: Infection, disease perceptions and drug uptake patterns in an endemic community after multiple rounds of mass drug administration. Parasites and Vectors. 2018;

13. Jones C, Ngasala B, Derua YA, Tarimo D, Reimer L, Bockarie M, et al. Lymphatic filariasis transmission in Rufiji District, southeastern Tanzania: infection status of the human population and mosquito vectors after twelve rounds of mass drug administration. Parasit Vectors. 2018;

14. NTDCP. Tanzania Lymphatic Filariasis Elimination Updates. unpublished; 2021.

15. Maritim P, Silumbwe A, Zulu JM, Sichone G, Michelo C. Health beliefs and health seeking behavior towards lymphatic filariasis morbidity management and disability prevention services in luangwa district, zambia: Community and provider perspectives. PLoS Negl Trop Dis [Internet]. 2021;15(2):114. Available from: http://dx.doi.org/10.1371/journal.pntd.0009075

16. Madon S, Olanya J, Ntuli M, Michael E. Social Science \& Medicine Can mobile phones help control neglected tropical diseases ? Experiences from Tanzania. Soc Sci Med [Internet]. 2014;102:103-10. Available from: http://dx.doi.org/10.1016/j.socscimed.2013.11.036

17. Kelly-hope LA, Blundell HJ, Macfarlane CL, Molyneux DH. Innovative Surveillance Strategies to Support the Elimination of Filariasis in Africa. Trends Parasitol [Internet]. 2018;34(8):694-711. Available from: https://doi.org/10.1016/j.pt.2018.05.004

18. Lester RT, van der Kop M, Taylor D, Alasaly K, Coleman J, Marra F. M-health: Connecting patients to improve population and public health. Vol. 53, British Columbia Medical Journal. 2011. p. 218-9.

19. Rashid AT. Mobile Phones and Development: An Analysis of IDRC-Supported Projects. 2014;(January 2009).

20. Stanton MC, Best A, Cliffe M, Kelly-Hope L, Biritwum NK, Batsa L, et al. Situational analysis of lymphatic filariasis morbidity in Ahanta West District of Ghana. Trop Med Int Heal. 2016;

21. Mwingira U, Chikawe M, Mandara WL, Mableson HE, Uisso C, Mremi I, et al. Lymphatic filariasis patient identification in a large urban area of Tanzania: An application of a community-led mHealth system. PLoS Negl Trop Dis. 2017;

22. Wattanapisit A, Amaek W, Wattanapisit S, Tuangratananon T, Wongsiri S, Pengkaew P. Challenges of implementing an mhealth application for personalized physical activity counselling in primary health care: A qualitative study. Int J Gen Med. 2021;14:3821-31. 
23. Mistry SK, Akter F, Yadav UN, Hossain MB, Sichel A, Labrique AB, et al. Factors associated with mobile phone usage to access maternal and child healthcare among women of urban slums in Dhaka, Bangladesh: A cross-sectional study. BMJ Open. 2021;11(4):1-10.

24. Woldie M, Feyissa GT, Admasu B, Hassen K, Mitchell K, Mayhew S, et al. Community health volunteers could help improve access to and use of essential health services by communities in LMICs: An umbrella review. Health Policy Plan. 2018;33(10):1128-43.

25. Krentel A, Gyapong M, Mallya S, Boadu NY, Amuyunzu-Nyamongo M, Stephens M, et al. Review of the factors influencing the motivation of community drug distributors towards the control and elimination of neglected tropical diseases (NTDs). PLoS Negl Trop Dis. 2017;11(12):1-23.

26. Lehmann U, Sanders D. Community Health Workers: What do we Know About Them? The State of Evidence on Programmes, Activities, Costs and Impact on Health Outcomes of Using Community Health Workers. 2007;(January).

27. Olaniran A, Smith H, Unkels R, Bar-Zeev S, van den Broek N. Who is a community health worker? - A systematic review of definitions. Glob Health Action [Internet]. 2017;10(1). Available from: http://dx.doi.org/10.1080/16549716.2017.1272223

28. WHO. Community health workers: A strategy to ensure access to primary health care services [Internet]. 2016. 37 p. Available from: https://apps.who.int/iris/handle/10665/249563

29. Macfarlane CL, Dean L, Thomson R, Garner P. Community drug distributors for mass drug administration in neglected tropical disease programmes: Systematic review and analysis of policy documents. J Glob Health. 2019;9(2).

30. Krentel A, Gyapong M, Mallya S, Boadu NY, Amuyunzu-nyamongo M, Stephens M, et al. Review of the factors influencing the motivation of community drug distributors towards the control and elimination of neglected tropical diseases (NTDs). 2017;1-23.

31. Debrah LB, Mohammed A, Osei-mensah J, Mubarik Y, Agbenyega O, Ayisi-boateng NK, et al. Morbidity management and surveillance of lymphatic filariasis disease and acute dermatolymphangioadenitis attacks using a mobile phone-based tool by community health volunteers in Ghana. 2020;1-17. Available from: http://dx.doi.org/10.1371/journal.pntd.0008839

32. Mwingira U, Chikawe M, Mandara WL, Mableson HE, Uisso C, Mremi I, et al. Lymphatic filariasis patient identification in a large urban area of Tanzania: An application of a community-led mHealth system. PLoS Negl Trop Dis. 2017;

33. Chaiyachati KH, Loveday M, Lorenz S, Lesh N, Larkan L, Cinti S, et al. A Pilot Study of an mHealth Application for Healthcare Workers: Poor Uptake Despite High Reported Acceptability at a Rural South African Community-Based MDR-TB Treatment Program. 2013;8(5):1-8.

34. Thondoo M, Strachan DL, Nakirunda M, Ndima S, Muiambo A, Källander K, et al. Potential roles of mhealth for community health workers: Formative research with end users in Uganda and Mozambique. JMIR mHealth uHealth. 2015;3(3).

35. Blanas DA, Ndiaye Y, MacFarlane M, Manga I, Siddiqui A, Velez O, et al. Health worker perceptions of integrating mobile phones into community case management of malaria in Saraya, Senegal. Int 
Health. 2015;7(3):176-82.

36. Soti DO, Kinoti SN, Omar AH, Logedi J, Mwendwa TK, Hirji Z, et al. Feasibility of an innovative electronic mobile system to assist health workers to collect accurate, complete and timely data in a malaria control programme in a remote setting in Kenya. Malar J. 2015;14(1):1-8.

37. Schuttner L, Sindano N, Theis M, Zue C, Joseph J, Chilengi R, et al. A mobile phone-based, community health worker program for referral, follow-up, and service outreach in rural Zambia: Outcomes and overview. Telemed e-Health. 2014;20(8):721-8.

38. Mc Kenna P, Babughirana G, Amponsah M, Egoeh SG, Banura E, Kanwagi R, et al. Mobile training and support (MOTS) service-using technology to increase Ebola preparedness of remotely-located community health workers (CHWs) in Sierra Leone. mHealth. 2019;5:35-35.

39. Kalinga AK, Ishengoma DS, Kavishe R, Temu L, Mswanya C, Mwanziva C, et al. The use of Fionet technology for external quality control of malaria rapid diagnostic tests and monitoring health workers' performance in rural military health facilities in Tanzania. PLoS One. 2018;13(12):1-18.

40. Long L, Pariyo G, Kallander K. Digital Technologies for Health Workforce Development in. Glob Heal Sci Pract. 2018;6(Supplement 1):1-8.

41. Andreatta P, Debpuur D, Danquah A, Perosky J. Using cell phones to collect postpartum hemorrhage outcome data in rural Ghana. Int J Gynecol Obstet. 2011;113(2):148-51.

42. Chang LW, Kagaayi J, Arem H, Nakigozi G, Ssempijja V, Serwadda D, et al. Impact of a mhealth intervention for peer health workers on AIDS care in rural Uganda: A mixed methods evaluation of a cluster-randomized trial. Vol. 15, AIDS and Behavior. 2011. p. 1776-84.

43. Chib A. The Aceh Besar midwives with mobile phones project: Design and evaluation perspectives using the information and communication technologies for healthcare development model. J Comput Commun. 2010;15(3):500-25.

44. DeRenzi B, Findlater L, Payne J, Birnbaum B, Mangilima J, Parikh T, et al. Improving community health worker performance through automated SMS. ACM Int Conf Proceeding Ser. 2012;25-34.

45. Ngabo F, Nguimfack J, Nwaigwe F, Mugeni C, Muhoza D, Wilson DR, et al. Designing and implementing an innovative SMS-based alert system (RapidSMS-MCH) to monitor pregnancy and reduce maternal and child deaths in Rwanda. Pan Afr Med J. 2012;13:1-15.

46. Agarwal S, Perry HB, Long LA, Labrique AB. Evidence on feasibility and effective use of mHealth strategies by frontline health workers in developing countries: Systematic review. Trop Med Int Heal. 2015;20(8):1003-14. 